\title{
Hirsutismo en niñas adolescentes. Estudio clínico y de laboratorio
}

\author{
Amanda Cortínez R.' : Ruth Schneider S.' ; Francisco Beas F.' ; \\ Martha Eggers M. ${ }^{1}$; Cecilia Henriquez del V. ${ }^{1}$
}

\section{Hirsutism in adolescent girls}

\begin{abstract}
Fourteen girls with hirsutism, aged 12.5 to 16 years, were prospectively st!Jdied. Patients with adrenal tumors, primary ovary disease or classical congenital adrenal hyperplasia were excluded from this studv. Hirsutism was evaluated by the Ferriman score. All patients had normal weight and blood pressure. Urinary 17 nydroxicorticos. teroids, pregnanetriol and dehydroepiandrosterone were measured in 24 hour urine samples. Lᄂ. FSH. cartisol, 17.hidraxyprosesterone (17-OH.P), dehydroepiandrosierone sulfate (DHEAS) and testosterone in plasme were also measured pefore and $60 \mathrm{~min}$ after ntravenous iniection of rapidly acting ACT! $\{0.25$ mg!. Ultrasonograpty of the ovary was also done to each patient. Two patients were probably heterozvgotus for 21-hydroxylase ceficiency. nine other garls showed high blood levels of DHEAS \{average $8289 \mathrm{ng} / \mathrm{ml}$ \} but in three cases no apparent cause was detected and they were labeled as idiopatic hirsutism. The two patients with evidence of heterozygosity for 21.hidroxylase deficiency and 5 patients with high DHEAS improved after dexamerasone treatment and normali. zation of their olood ievels of $17-0 \mathrm{HP}$ and DHEA-S respectively.
\end{abstract}

(Key words: hirsu-ism, girls, Edolescent, ACTH, LH. FSH, cortisol, LHEAS, 17-CHP, testosterone.)

Se denomina hirsutismo al aumento del vello corporal en zonas andrógeno dependientes: supralabial, barbilla, mejillas, línea media torá cica y abdominal, aréola mamaria, glúteos o cara interna de los muslos. Provoca serios problemas psicológicos en las mujeres, especialmente las adolescentes, en quienes, con cierta frecuencia, se asocia a trastornos menstruales, obesidad e infertilidad", en una etapa del desarrollo en que la "imagen corporal" y la "interacción con sus pares" son factores críticos en el desarrollo de la autoestima y de la personalidad. El hirsutismo puedc clasificarse en exógeno (administración de medicamentos con acción androgénica), ovà. rico (síndrome de ovario poliquístico - SOP -- y tumores ováricos), suprarrenal (tumor e hiperpla. sias suprarrenales virilizantes) y de causa desconocida o idiopático (aquellos en que se descartan las causas anteriores y las determinaciones hormonales resultan normales en sangre $y$ orina).

En el hirsutismo de origen suprarrenal se han descrito deficiencias parciales de las enzimas 21 -hidroxilasa $(21-\mathrm{OH}), 11$-hidroxilasa $(11-\mathrm{OH}) \mathrm{y}$ $3 \beta$-hidroxiesteroide dehidrogenasa (3 $\beta$-HSD)

1. Instituto de Investigaciones Materno-Infanti\}, Facultad de Medicina, Universidad de Clile, Hospital Clínico San Borja-Arriarán. que no se manifiestan en recién nacidos o en los primeros años de vida, sino más tarde, especialmente cerca de la pubertad o en adultos, provocando hiperandrogenismo e hirsutismo ${ }^{2-11}$. Estos trastornos pueden confundirse con cierta frecuencia con el síndrome de ovario poliquístico porque, además de signos clínicos de hiperandrogenismo, la ecografía ovárica revela a veces imágenes quísticas similares en ambas enfermedades ${ }^{12,13}$. Sólo un acucioso estudio hormonal en condiciones basales, con pruebas de estimulación con ACTH y luego frenación, permiten en la mayoría de los casos el diagnóstico correcto $6, ?, 14,15$. A continuación se describe una estrategia realizada para detectar, en un grupo de pacientes con hirsutismo de etiología no precisada, aquellas en que la causa puede ser de origen suprarrenal y sus resultados.

\section{Material y Método}

Sc extudiaron prospectivamente 14 niñas de 12 años 6 meses a 16 ẫos de edad que sutín jan hirsutismo y fuesen normotensas, de peso y estatura dentro del rango normal para la población chilena. Se descartaron las que tenían tumor o hiperplasia suprarrenal clásica, enfermedades ováricas o concentraciones anormales de gonadotrofinas séricas. Fn todas las pacientes se realizó anamnesis completa, investigándose dirigidamente ante- 
cedentes familiares de hirsutismo, fecha de aparición de éste, drogas que pudieran afectar el crecimiento del vello y trastarnos menstruales, además se les hizo examen físico completo incluyendo antropomet tía y examen ginecológico, que se complementó con ecotomografía pelviana. Il hirsutismo se calificó en escala de 1 a 36 según la puntuación de Ferriman ${ }^{36}$.

Para analizar y describir el resultado de las ecograf'ás se definió como ovario normal (ON) al que medía hasta 6 cc de volumen $y$ sus foliculos eran menores dc $4 \mathrm{~mm}$ : ovario poiquístico (OPU) el que medía más de $8 \mathrm{cc}$, tenía cápsula gruesa e inágenes econegativas múltiples; se llamó ovario micropoliquístico (MPQ) a la situación intermedia entre las 2 anteriores (entre $6 y$ $8 \mathrm{cc}$, folículos múltiples pero pequeños). Esta clasificación se hizo con el fín de observar la evolución de las ecografías de cada paciente $y$ no tiene valor diagnóstico ni pronóstico.

El estudio endocrinológico de cada paciente se hizo con mediciones basales de esteroides en orina de 24 horas en dos oportunidades, incluyendo 17 ketosteroides (17 KS), 17-hidroxicorticosteroides (17 OHC), pregnanetriol (PNT) y dehióroepiandrosterona (DHEA): posteriormente se practicó prueba ACTH rápida (en el curso de los primeros 8 días del ciclo menstrual, en ayunas, entre las 8 y 10 horas, se extrajo sange basal para determinar cortisol (F), 17-hidroxiprogesterona (17-OHP), sulfato de dehidroepiandrosterona (DHEA-S) y testosterona (T). Luego se inyectaron, por váa endovenosa. $25 \mu \mathrm{g}$ de 1.24 ACTH. y 60 minutos después se tomó nueva muestra para determinar los mismos esteroides!.
Las pacientes cuyos exámenes sugerian deföciencia enzimática suprartenal: 17-OHP o DHEA-S tasal elevados $y, 0,17-0 H P$ aumentada después del empleo de ACTH, sobre lo esperado para la pobiación normal (de acuerdo al monograma de $\mathrm{New})^{18}$, fueron tratadas con dexametasona $0,5 \mathrm{mg}$ oral al acostarse. Después de iniciado este tratamiento se cvaluaron clínicamente cada 3 meses. cada 6 meses se repitieron todas las determinaciones de esteroides plasmáticos y urinarios y, finalmente, se efectuó una ecografia ovárica de control al completar un año de tratamiento ininterrumpido.

Las mediciones de li, 1?-OHP, DHEA-S y $T$ se hicieron por RIA con isótopo marcido con Tritio (New England Nuclear Boston, Massachusetts), los análisis de cada muestra se hicieron por duplicado con coeficientes de variación intra e interensayo menores de $10 \%$. Los 17 KS y el 17 OHC fueron determinados por el metodo de Zimmerman y el PNT y DHEA-S mediante cromatografía en capa fina.

\section{Resultados}

De acuerdo con los resultados obtenidos en cada caso, las pacientes pudieron agruparse en nueve con DHEA-S elevado, dos probables portadoras heterozigotas del gen de deficiencia de la 21 hidroxilasa y tres niffas con hirsutismo idiopatico.

Pacientes con DHEA-S elevado. Sus manifestaciones clínicas se resumen en la tabla 1 . Los

Tabla 1

Característjcas clínicas de las pacientes con hirsutismo

\begin{tabular}{|c|c|c|c|c|c|c|c|}
\hline \multirow[b]{2}{*}{ Nombre } & \multirow[b]{2}{*}{$\begin{array}{l}\text { Edad } \\
\text { eronológica } \\
\text { (años) }\end{array}$} & \multicolumn{3}{|c|}{ HIRSUTISMO } & \multicolumn{3}{|c|}{ MENSTRUACIONES } \\
\hline & & $\begin{array}{l}\text { Antecedente } \\
\text { familiar }\end{array}$ & $\begin{array}{l}\text { Edad } \\
\text { aparición } \\
\text { (años) }\end{array}$ & $\begin{array}{l}\text { Puntos } \\
\text { (Ferriman) }\end{array}$ & $\begin{array}{l}\text { Menarquia } \\
\text { (años) }\end{array}$ & $\begin{array}{l}\text { Caracteristicas } \\
\text { clínicas }\end{array}$ & Acné \\
\hline \multicolumn{8}{|l|}{1} \\
\hline E.N.R. & 13 a $6 \mathrm{~m}$ & - & 10 & 6 & 12 & Normales & ++ \\
\hline J.M.S. & 16 & & 12 & 9 & 11 & Nomales & +++ \\
\hline S.C.G. & 13 a $6 \mathrm{~m}$ & - & 7 & 12 & 11 & No rmales & ++ \\
\hline J.T.N. & 15 & + & $s$ & 8 & 12 & Normales & - \\
\hline R.R.P. & 17 a $6 \mathrm{~m}$ & + & 12 & 12 & 12 & Normales & ++ \\
\hline M.G.P. & 15 & . & 10 & 8 & 13 & Normales & ++ \\
\hline N.T.B. & & + & $12 *$ & 14 & 12 & Normales & + \\
\hline M.V.A. & 15 & + & 13 & 9 & 11 a $10 \mathrm{~m}$ & Nomales & + \\
\hline M.P.R. & 14 a $6 \mathrm{~m}$ & + & 11 & 8 & 12 & Nomnales & ++ \\
\hline \multicolumn{8}{|l|}{ II } \\
\hline C.G.A. & 13 & $\star$ & $11 \mathrm{a} 6 \mathrm{~m}$ & 13 & 11 a $10 \mathrm{~m}$ & Oligomenorrea & ++ \\
\hline X.C.P. & 14 a $6 \mathrm{~m}$ & - & 12 a $6 \mathrm{~m}$ & 6 & 13 & Oligomenorrea & ++ \\
\hline \multicolumn{8}{|l|}{ HI } \\
\hline P.E.P. & 12 a $6 \mathrm{~m}$ & + & $12 \mathrm{a} 6 \mathrm{~m}$ & 6 & 11 a $8 \mathrm{~m}$ & Nomnales & No \\
\hline М.Н.В. & 13 a $6 \mathrm{~m}$ & + & 9 & 16 & 11 a $6 \mathrm{~m}$ & Normales & $\mathrm{No}$ \\
\hline C.U.L. & 8 & + & R.N. & 10 & & - & No \\
\hline
\end{tabular}

*: Hipertricosis desde el nacimiento, que evolucionó a hirsutisno a los 12 años.

I: Pacientes con DHEA-S elevado.

II: Pacientes heterozigotas para el gen de deficiencia de la 21-hidroxilasa.

III: Hirsutismo aparentemente idiopático. 
$17 \mathrm{KS}$ urinarios estaban elevados sobre $12 \mathrm{mg}$ en $24 \mathrm{~h}$ en tres pacientes; en las seis restantes eran normales. Los otros esteroides urinarios (17 OHC, PNT y DHEA) estaban en cantidad normal en todas las niñas. En el plasma los esteroides $F, 17-0 H P$ y $T$ eran nomales; sin embargo, la DHEA-S basal estaba francamente elevada en todas ellas (promedio $8216 \mathrm{ng} / \mathrm{ml}$, variando de 4750 a $14800 \mathrm{ng} / \mathrm{ml}$ ). Después del estímulo con ACTH no se observaron cambios significativos en 17-OHP ni DHEAS (tabla 2).

Sólo cinco de estas pacientes fueron tratadas (las restantes no continuaron control) con dexametasona $0,5 \mathrm{mg}$ oral al acostarse; en todas ellas DHEA-S descendió rápidamente a valores dentro del rango normal, lo gue descartaba un tumor suprarrenal; sólo en tres el tratamiento se prolongó por mâs de un año, disminuyendo el hirsutismo y el acné, como también mejorando, en dos de ellas, las imágenes de la ecotomografia ováriça (tabla 3).
Pacientes probables portadoras heterozigotas del gen de deficiencia de la 21 -hidroxilasa. El cuadro clínico de ambas se resume en la tabla 1. En la niña CGA el contenido de $17 \mathrm{KS}$ y PNT urinarios, asi como las concentraciones plasmá. ticas de LH, FSH y esteroides (F, 17-OHP y DHEAS) eran nonmales en múltiples oportunidades. La tasa de 17-OHP se le encontró aumentada en dos de siete mediciones $(2,6$ y 4,68 $\mathrm{ng} / \mathrm{ml}$ ) (tabla 2) y no aumentó en la prueba de ACTH rápido. Actualmente la paciente ha completado 4 años de tratamiento con dexametasona $0,5 \mathrm{mg}$ oral al costarse, le han disminuido el acné y el vello, sus menstruaciones y la ecografía ovárica son normales (tabla 3).

En la paciente XCP los esteroides urinarios, LH, FSH, 17-OHP, DHEA-S y $F$ en plasma fue. ron normales, la ecotomografía ovárica mostraba ovarios poliquísticos (tabla 2). Se inició terapia con dexametasona $0,5 \mathrm{mg}$ al acostarse, observándose nomalización de la 17-OHP -a $0,6 \mathrm{ng} / \mathrm{ml}-\mathrm{y}$ de sus menstruaciones. Ha com-

Tabla 2

Parámetros hormonales basales y 60 minutos postest ímulo con ACTH $0,25 \mathrm{mg}$ e.v.

\begin{tabular}{|c|c|c|c|c|c|c|c|c|c|c|}
\hline \multirow[t]{2}{*}{ Nonibre } & \multirow{2}{*}{$\begin{array}{l}17 \mathrm{KS} \mathrm{mg} \text { ! } \\
\text { orina } 24 \mathrm{~h}\end{array}$} & \multicolumn{2}{|c|}{ Cortisol ng/ml } & \multicolumn{3}{|c|}{ 17-OHP ng/mt } & \multicolumn{2}{|c|}{ DHEA-S ng/ml } & \multirow{2}{*}{$\begin{array}{c}\text { T ng/ml } \\
\text { Basal }\end{array}$} & \multirow{2}{*}{$\begin{array}{l}\text { Ecotonlog. } \\
\text { pelviama }\end{array}$} \\
\hline & & Basal & Post ACTH & Basal & Pos & ACTH & Basal & Post ACTH & & \\
\hline \multicolumn{11}{|l|}{ l } \\
\hline E.N.R. & 20,6 & 160 & 360 & 0.96 & & 2,39 & 7200 & 14000 & 0,60 & M.P.Q \\
\hline J.M.S. & $7 \sqrt{5}$ & 120 & 290 & 0,56 & & 1,36 & 11000 & 9800 & 0,40 & Normal \\
\hline S.C.G. & 8,8 & 130 & 300 & 0.32 & & 1.33 & 9200 & 7700 & 0,51 & O.P.Q. \\
\hline J.T.N. & $\lfloor 3,1$ & 250 & 440 & 1,70 & & 1,10 & 4750 & 6000 & - & Normal \\
\hline R.R.P. & 15,3 & 70 & 400 & 0,68 & & 1,88 & 5100 & 6100 & 0,53 & O.P.Q. \\
\hline M.G.P. & 9,6 & 90 & 150 & 0,67 & & 2,30 & 7500 & 6000 & - & Normal \\
\hline N.T.B. & 9,6 & 160 & 320 & 0,78 & & 2,50 & 14800 & 11600 & 0,40 & Normal \\
\hline M.V.A. & 10,1 & 190 & 315 & 2,92 & & 3,97 & 7600 & 5500 & 0,63 & O.P.Q \\
\hline M.P.R. & 8,2 & 210 & 260 & $\mathbf{1}, 98$ & & 1.69 & 7800 & 7900 & 0,40 & Normal \\
\hline Promedio & 12,5 & 153 & 315 & 1,17 & & 2.05 & 8328 & $82 B 9$ & 0,49 & \\
\hline \multicolumn{11}{|l|}{ II } \\
\hline C.G.A. ${ }^{*}$ & 11.7 & 200 & - & 2,14 a & 4,68 & 2,37 & 3200 & - & 0,80 & O.P.Q. \\
\hline X.C.P. & 12,5 & 160 & 270 & 1,17 & & 5,38 & 3350 & 3900 & & O.P.Q. \\
\hline \multicolumn{11}{|l|}{ III } \\
\hline P.E.P. & 7,0 & 110 & 300 & 0,64 & & 3,10 & 2175 & 1575 & - & M.P.Q \\
\hline M.H.B. & 6.4 & 15 & 240 & 0.27 & & 1,50 & 1050 & 1225 & 0,20 & Normal \\
\hline C.U.L. & 3,15 & 200 & 320 & 0,35 & & 1,08 & 2100 & 2350 & 0,09 & Normal \\
\hline
\end{tabular}

Nota: Valores normales para nuestro laboratorio: $17 \mathrm{Ks}=$ hasta $13 \mathrm{mg} / 24 \mathrm{~h}$; cortisol basal=50 a $250 \mathrm{ng} / \mathrm{ml}$; post $\mathrm{ACTH}=$ aumento doble de la basal; 1 ? -OH progesterona basal=hasta $1.9 \mathrm{ng} / \mathrm{ml}$; 60 ' post $\mathrm{ACTH}=3.5 \pm 2,2 \mathrm{ng} / \mathrm{ml}$, o un $1,98 \pm 0,96 \mathrm{ng} / \mathrm{ml}$ ( $\left(M . \mathrm{l}\right.$. New $\left.{ }^{13}\right)$; testosterona=menor $0,6 \mathrm{ng} / \mathrm{ml} ; D H E A-S$ basal=hasta $3600 \mathrm{ng} / \mathrm{m}$.

*: Pacicnte C.G A. se trato con dexametasona $0,5 \mathrm{mg}$ al acostarse durante un afio; la prueba con $\mathrm{ACTH}$ se practicó luego de suspendido un mes este tratamiento.

l: Pacientes con DHEA-S elevado.

II: Pacientes probables heterozigotas para el gen de deficiencia de la 21-hidroxilasa.

III: Hirsutismo aparentemente idiopático.

$17 \mathrm{KS}: 17$ ketoest eroides. 17-OHP: 17 hidroxiprogesterona. DHEA-S: dehidroepiandrosterona sulfato.

T: testosterona. 
Tabla 3

Respuesta al tratamiento con dexametasona $(0,5 \mathrm{mg}$ al acostarse $)$ en 7 pacientes con hirsulismo

\begin{tabular}{|c|c|c|c|c|c|c|c|c|c|}
\hline \multirow[t]{2}{*}{ Sombre } & \multicolumn{2}{|c|}{ Puntos (Ferriman) } & \multicolumn{2}{|c|}{ Menstruaciones } & \multicolumn{2}{|c|}{ DHEA-S ng/ml } & \multicolumn{2}{|c|}{ Ecotomograf ía } & \multirow{2}{*}{$\begin{array}{c}\text { Duración del } \\
\text { tratamiento }\end{array}$} \\
\hline & B & $\mathrm{CT}$ & B & $\mathrm{C} / \mathbf{T}$ & B & $C / \mathbf{T}$ & $\mathrm{B}$ & $\mathrm{C} / \mathrm{T}$ & \\
\hline \multicolumn{10}{|l|}{ ] } \\
\hline E.N.R. & 6 & 4 & $\mathrm{~N}$ & $x$ & $? 200$ & 2550 & M.P.Q. & Normal & 2 a $4 \mathrm{~m}$ (intermitente) \\
\hline S.C.G. & 12 & 9 & $\mathbf{N}$ & $\mathrm{N}$ & 9200 & 4200 & P.P.Q. & & I a (irregular) \\
\hline R.R.P. & 12 & 9 & $\mathrm{~N}$ & $\mathbf{N}$ & 5100 & 203 & O.P.Q. & M.P.Q. & 1 a $4 \mathrm{~m}$ \\
\hline N.T.B. & 14 & 10 & $\mathrm{~N}$ & $N$ & 14800 & 2075 & Normal & & 1 a $3 \mathrm{~m}$ \\
\hline M.V.A. & 9 & 9 & $\mathrm{~N}$ & $\mathbf{N}$ & 7600 & 2950 & O.P.Q. & - & 3 semanas \\
\hline \multicolumn{10}{|l|}{ II } \\
\hline C.G.A. & 13 & 10 & $\begin{array}{l}\text { Oligo- } \\
\text { meno- } \\
\text { trea }\end{array}$ & $\mathrm{N}$ & 2950 & 555 & O.P.Q. & Normal & $4 \mathrm{a}$ \\
\hline X.C.P. & 6 & 5 & $\begin{array}{l}\text { Oligo- } \\
\text { meno- } \\
\text { rrea }\end{array}$ & $\mathrm{N}$ & 3300 & 600 & O.P.Q. & & $8 m$ \\
\hline
\end{tabular}

B: Basal. C/T: con tratamicnto. N: nomal, O.P.Q.: Ovario poliquístico. M.P.Q.: ovario mjctopoliqu ístico.

I: Pacientes con DHEA-S elevado.

II: Pacientes probables heterozigotas para el gen de deficiencia de la 21 -hidroxilasa.

pletado 17 meses de tratamiento, le han disminuido el acné y el vello (tabla 3 ).

Pacientes con hirsutismo idiopático. En estas tres niñas (tabla 1) el hirsutismo apareció a una edad muy variable, su intensidad varió de 6 a 16 según la calificación de Ferriman y en dos casos el vello tenía distribución androide. No presentaban trastornos menstruales, eran normotensas, de peso y estatura adecuados, sin acné o alteraciones de genitales extemos. FSH y LH plasmáticos, como también los esteroides urinarios y plasmáticos estudiados eran normales, en condiciones basales y después del estimulo con ACTH (tabla 2), por lo que no puede suponérseles causas ováricas o suprarrenales.

\section{Comentario}

La distribución de los trastornos causales de hirsutismo en esta serie concuerda con la publicada por otros, que, entre 52 mujeres hirsutas, encontraron en 59\% elevación de la DHEA-S únicamente o en asociación con aumento de la testosterona total o libre, $11 \%$ sin alteraciones de ningún resultado y $30 \%$ con aumentos de dj. fícil interpretación - de la concentración de otros esteroides en la sangre $\mathbf{u}$ orina ${ }^{17}$. La respuesta al estímulo con ACTH de acción rápida en las pacientes catalogadas como probables portadoras heterozigotas fue, en una de ellas, me- nor que el esperado en casos de deficiencia no clásica de 21-hidroxilasa, si bien compatible can el de portadora heterozigota del gen de deficiencia de la enzima de acuerdo al nomogra. ma de New y cols. ${ }^{18}$. La falta de aumento de 17-0HP sobre la basal después del estímulo con ACTH en la segunda de ellas fue atribuida a una prolongada y reciente frenación con dexameta. sona. Los heterozigotos de enfermedades autosómicas recesivas pueden presentar, en efecto, algunos sintomas, probablemente como resultado de deficiencia enzimática parcial. Zachmann y col. han publicado tres pacientes similares, denominandolos "heterozigotos inusuales" de hiperplasia suprarrenal por deficiencia de 21 hidroxilasa ${ }^{19,20}$.

En las pacientes con hirsutismo, grados variables de acné, ciclos menstruales normales y concentraciones elevadas de DHEA-S, la norma. lización de la concentración de esta ủltima con dexametasona hace improbable un tumor. La DHEA-S elevada es frecuente en pacientes con hirsutismo, acné $y$ ciclos menstruales normales ${ }^{1 ?}$, pero también puede estar aumentada en mujeres sin hirsutismo, con infertilidad primaria o secundaria, oligomenorrea u ovario poliquistico ${ }^{21}$. La ocurrencia de casos con o sin hirsutismo entre pacientes con aumento de la concentración de DHEA-S se explicaría por el poco efecto virilizante de la hormona y la amplia variación indi- 
vidual, genéticamente determinada, de la sen. sibilidad a los andrógenos de los receptores correspondientes ${ }^{20}$. Las concentraciones altas de DHIEA-S indican aumento de la actividad suprarrenal $^{21,22}$, pero no su etiología, que puede ser el estrés de un paciente infértil' ${ }^{17}$, alteraciones del metabolismo de la prolactina $y$ bloqueos enzimáticos parciales ${ }^{17,19,23,24}$.

La mejoría clínica del hirsutismo en las pa. cientes tratadas con dexametasona debe ser interpretada con cautela, pues si bien parece tentador atribuir la disminución del vello a una deficiencia enzimática suprarrenal parcial, la dexametasona ejerce también acciones en otros niveles, ya que eleva el nivel de la globulina transportadora de hormonas sexuales (SHBG) ${ }^{24}$ a la que $80 \%$ de la testosterona circula unida, en circunstancias que sólo las fracciones libre $y$ unida a albúmina son biologicamente activas. pudiendo, por lo tanto, un aumento de la SHBG provocar disminución del hirsutismo. Se ha señalado también que la dexametasona mejora el hirsutismo que se acompaña de hiperprolactinemia ${ }^{23,24}$. Estudios clínicos con catcterización simultánea de la vena ovática y adrenal ${ }^{25}$ sugieren que la hiperproducción de andrógenos observada en pacientes con ovario poliquistico es de origen ovárico, pero también puede ser suprimido por la dexametasona.

En resumen, cierta proporción de las pacientes con hirsutismo antiguamente catalogado de idiopático, pueden ser portadoras heterozigotas del gen de deficiencia de la 21 hidroxilasa o mostrar aumento de la DHEA.S que requiere mayor estudio. En la medida que la exploración funcional pueda ser aplicada corrientemente en estos casos, el diagnóstico será más preciso y el tratamiento mejor fundamentado.

\section{Resumen}

Se estudiaron prospectivamente 14 nitias con hirsutismo, entre los 12.5 y 16 años de edad, normotensas, de peso corporal normal, sin tumores, afecciones ováricas primarias o hiperplasia suprarrenal clásica. El hirsutismo se calificó según Ferriman. Se hicieron determinaciones basales de 17 ketosteroides, 17 hidroxicorticoides, pregnanetriol y dehidroepiandrosterona en orina de 24 horas, así como de L.H, FSH, testusterona, cortisol (F), 17-hidroxiprogesterona (17-OHP), dehidroepiandrosterona-sulfato
(DHEA.S) en plasma, mediciones de las tres úl. timas 60 min después de inyectar por via endovenosa $0,25 \mathrm{mg}$ de corticotrofina de acción rápida y ultrasonografía ovárica. De acuerdo al cuadro clínico y de laboratorio dos de las pa. cientes eran probables portadoras heterozigotas del gen de deficiencia de 21 hidroxilasa, nueve sin trastornos menstruales tenían DHEA-S basal elevada (promedio 8289 , márgenes 4750 a $14800 \mathrm{ng} / \mathrm{ml}$ ) como único hallazgo de laboratorio y su hirsutismo era, por lo tanto, de etiología probablemente suprarrenal, estando aún en estudio para el diagnóstico definitivo. Finalmente, las otras tres pacientes tienen hirsutismo idiopático. Las dos niñas probablemente portadoras heterozigotas del gen de deficiencia de la $21-\mathrm{OH}$ y cinco de las que tenían la DHE $A-S$ elevada, fueron tratadas con dexametasona, con normalización de la DHEA-S, mejoria del aspecto ecográfico ovárico y del hirsutismo.

(Palabras clave: hirsutismo, niñas adolescentes, ACTH, LH, FSH, cortisol, DHEA-S, 17 OHP, testosterona.)

\section{Referencjas}

I. Kustin $J$ and Rebar $R$ : Hirsutism in young adolescent girls. Pediatr Ann 1986: 522-528.

2. Miller Watrer and Levine Leonore: Molecular and clinical advances in congenital adrenal hyperplasia. J Pediatr 1987: 111:1-13.

3. Khon $B$, Levine $L$, Pollack $W$ et al: Late onset steroid 2I hidroxylase deficiency: A variant of classical congenital adrenal hyperplasia. J Clin Vindocrinol Metab 1983:55: 817-822

4. Knorr D. Bidlignmater F, Holler $W$ et al: Is heterozigosity for the steroid $21-$ hydroxylase deliciency responsible for hirsutistn, premature pubarche. early puberty and precocious puberty in children? Acta Endocrinol Suppl (Copenli) 1986: 279 : 284-287.

5. Chetkowski $R J$, De Fazio L, Shomonki f et at. The incidence of late-onset congenital adrenal hyperplasia due to 2l-hydroxilace deficiency among hirsute women. J Clin Endocrinol Metab $1984 ; 58: 595-598$.

6. Fiet J. Gueux B. Gourmelen $M$ et al.: Comparison of basal and adrenocorticotropin plasma 21deoxycortisol and 17-Jydroxyprogesterone values as biological markers of late-onset adsenal hyperplasia. I Clin Endocrinol Metab 1988; 66: 659 . 667.

7. Azzis $R$ wha Zacur F.A: 21-hydroxilase deficitncy in temale hypcrandrogenic: screening and diagnosis. $J$ Clin Endocrinol Metab 1989; 69: 577-584.

8. Miller $\boldsymbol{W}$, and Levine L.: Molecula and clinical advances in congenital adrenal hyperplasia. J Pediatr 1987; $111:$ :-13. 
9. Guthrie GP, Emery WA, Quillén DL er al.: Adrenal androgen excess and defective 11-hydroxylation in women with idiopatic hirsutisn. Arch Intern Med 1982; 142: ?29-733

10. Pang $S$. Lemer $A$. Stoner $E$ et al: Late onset adrenal steroid 3-hidroxysteroid dehydrogenase deficiency. A cause of hirsutism in pubertal and postpubertal women. J Clin Endocrinol Metab L985: 60: 428-438.

11. Rosenfield $R$, Rich $B$, Walfolorf $J$ ef al.: Pubertal presentation of congenital $3 \beta$ hydroxysteroid dehydrogenase deficiencia. J Clin Endocrinal Metab $1980 ; 51: 345-353$.

12. Yen SSC: The polycistic ovary syndrome. Clin Endocrinol 1980;12:177-181.

13. Lobo R. Goehelsmonn VI and Horton $R$ : Evidence for the importance of peripheral tissue events in the development of hirsutism in Polycistic ovary syndrome. J Clin Endocrinol Metab 1983;57: 393 . 397.

14. Goumelen M, Phom-Hut.Trung MT, Bredon MG and Girard F: 17-Hydroxyprogesterone in the cosyntropin test: Results in normal and hirsute women and in mild congenital adrenal hyperplasia. Acta Endocrinol 1979:90: 481-489.

15. Gibson $M$, Locktits $R$ and Schiff $I$ : Abnotrial adrenal response to adrenocorticotropic hormone in hyperandrogenic women. Fertil Steri] 1980; $33: 43-48$.

16. Ferriman D. Gallway $7 D$. Chinical assessment of body hair growth in women. I Clin Endocrinol Metab 1961:21: 14413-1447.

17. Lobo $R$, Willington $L$ and Goebelsman V: Scrum levels of DHEA-S in gynecologic endocrinopathy and infertility. Obstet Gynccol 1981; 57. 607. 611

18. New MI: Genotyping steroid 21-hydroxylase deficiency. Hormonal reference data. J Clin Endocrinol Metab 1983; 57: 320-324.
19. Zachmann M. Prader A: Unusual lieterozygotes of congenital adrenal hyperplasia due to 21-hydroxylase deficiency. Acta Endocrinol (Copenh) 1978:87:557.

20. Zachmann M, Praler A: Unusual heterozygotes of congenital adrenal hyperplasia due to 21-liydroxylase deficiency confirmed by HLA tissue typing. Acta Endocrinol (Copent) 1979: 92: 542.

21. Ayes J: Differential response to adrecorticotropin hormone stimulation in polycystic ovarian disease with high and low dehydroepiandrosterone sulfatc levels. Fertil Steril $1982 ; 37: 645-649$.

22. Bailey-Pridham $D$, and San Filippo $T$ : Hrsutism it the adolescent female. Pediatr Clin North Am $1989 ; 36: 581 \cdot 599$.

23. Lobo $R$, Pal $W$ and Goebelsman $V$ : Dehydroepiandrosterone sulfate as an indicator of adrenal androgen function. Obstet Gynecol 1981; 57:69-72.

24. Korth-Schutz $S$, Levine $L$ and New $M$ : Dehydroepiandrosterone sulfate, a rapid test of abnormal adrenal androgen secretion. J Clin Findocrinol Metab 1976; 42: 1005-1013.

25. Corter JN. Tyson JE. Worne GL: Adrenocortical function in hyperprolactinemic women. J Clin Lindocrinol Metab 1977; 45:973-976.

26. Glickmas SP, Rosenficld $R L$, Bergenstal $R M$ et al.: Multiple androgenic abnomalities including elevated free testosterone in hyperprolactinemic women. J Clin Lindocrinol Metdb 1982: 55: 251257.

27. Waichenbers BL, Achondo SS, Okada $H$ ef al.: Determination of the source(s) of androgen, overproduction in hirsutism aswocjated with polycistic ovary syndrome by simultaneous adrenal and ovarian catheterization: Comparison with the dexametasone supression test. J Clin Endocrinol Metab $1986 ; 63: 1204-1210$.

\section{A YISO A LOS AUTORES}

Por acuerdo del Comité Editorial, la Revista Chilena de Pediatría devolverá sin tramitar todos los trabajos que no den estricto cumplimiento al Reglamento de Publicaciones, que se edita en cada número, y a las Instrucciones a los Autores, que aparecen en los números 3 y 6 de cada volumen desde 1986. 